\title{
Encore de nouvelles lignes directrices !
}

\author{
Roger LADOUCEUR
}

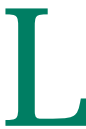

es lignes directrices que l'on nomme aussi « guides de pratique » (le terme « guidelines » est utilisé en anglais) sont des recommandations dével oppées de façon systématiquepar des experts pour ai der les médecins et les patients à prendre des décisions appropriées à propos de la santé. Elles jouissent, en médecine, d'une grande popularité Elles apparaissent comme une façon pratique de s'y retrouver dans l'immensité des connaissances médical es et une solution simple pour éviter les pratiques médicales déviantes. On prétend qu'elles peuvent aider à améliorer la qualité, la pertinence et les coûts des soins de santé. O n croit aussi qu'elles constituent d'excellents outils en éducation². Elles jouissent d'un accueil favorable et d'une grande crédibilité : il suffit qu'un collègue invoque les recommandations d'une association ou d'un groupe de travail en affirmant : " selon telles lignes directrices, il faudrait utiliser ce test ou ce médicament... » ou « selon les recommandations de tel groupe de travail, il serait préférable de faire ceci ou cela... », pour que la plupart ait tendance à adhérer à ce qui est suggéré de faire. Les lignes directrices jouissent donc en médecine d'un statut particulier et c'est pourquoi, sans doute, elles sont $s$ nombreuses.

\section{Mais, avonsnous raison de leur faire ains confiance?}

Car, après tout, les lignes directrices ne sont pas sans reproche. L'un des problèmes tient certainement à leur multitude. Juste pour l'hypertension artérielle, nous disposons actuellement d'au moins quatre grandes lignes directrices : les recommandations de l'O rganisation mondiale de la santé, les recommandations américaines (J N C $\mathrm{VI}$ ), les canadiennes et les québécoises. N emanquent plus que les régionales et les municipales! Pour la plupart des maladies fréquentes, il est facile de répertorier de 2 à 3 guides de pratique différents. $\mathrm{H}$ ibble et coll. ${ }^{3}$ ont d'ailleurs illustré l'absurdité de cette situation en compta bilisant toutes celles qu'ils pouvaient trouver : ils ont réussi à répertorier 855 lignes directrices différentes! Avec flegme, ces auteurs britanniques ont empilé toutes ces recommandations et ont noté qu'elles faisaient 27 pouces de haut, justifiant le titre de leur article: «Guidelines in general practice: the new Tower of Babel ». Ils ont surtout remarqué que les lignes directrices se développaient à un rythme infernal : 8 en 1990, 73 en 1995 et 138 en 1996. N ous sommes donc littéral ement inondés de lignes directrices, et il semble bien que ce flot (fléau ?) ira en augmentant au cours des prochaines années.

Un autre problème avec les lignes directrices vient aussi de leur crédibilité S'il en existe d'excellentes qui répertorient et analysent tous les travaux existants et émettent des recommandations basées sur une anal yse rigoureuse des preuves scientifiques, il y en a beaucoup d'autres de piètre qualité. Par exemple, celles formulées uniquement à partir d'opinions d'experts; ou celles produites par certaines associations professionnelles ou groupes de pairs qui donnent l'impression qu'il n'y a que leur problématique qui compte; ou encore celles réal isées sous l'égide de compagnies pharmaceutiques ! C es recommandations servent évidemment l'intérêt de ceux qui les produisent puisqu'ils permettent de sensibiliser les médecins et la population à l'importance de la maladie et facilitent l'attribution des budgets de santé et de recherche. C'est un vieux truc de marketing : plus on en parle, plus on y pense. La publication de lignes directrices devient un puissant véhicule de mobilisation créé par le consensus. Cette question de cré dibilité des lignes directrices a d'ailleurs fait l'objet d'une publication récente: "A re Guidelines following Guidelines $»^{4} D$ ans cette recherche, Shaneyfelt et coll. se sont demandé si les lignes directrices publiées dans des revues révisées par des pairs respectai ent les standards méthodologiques reconnus. Ils ont découvert qu'à peine $43 \%$ se conformaient aux exigences requises. En somme, s'il existe quelques 855 lignes directrices comme le démontre H ibble et que seulement 279 ont été répertoriées dans cette étude, on juge que moins de la moitié sont

Correspondance : Dr Roger Ladouceur - Département de médecine de famille - Faculté de médecine

Université de Montréal - CP 6128 - Succursale centre ville - Montréal, Québec - Canada H3C 3J7

Courriel : rladouceur@ssss.gouv.qc.ca 
correctes, c'est dire qu'environ $15 \%$ des lignes directrices sont correctes ! Voilà, certes, de quoi déchanter à leur sujet.

Ce n'est pas tout, les lignes directrices ont un autre problème: elles changent souvent d'avis. Prenons le dépistage du cancer du col utérin : auparavant les lignes directrices recommandaient de faire un frottis cervical chaque année jusqu'à ce que ce que les recommandations statuent pour un test tous lestrois ans. Considéronsmaintenant ledépistage du cancer de la prostate : à l'époque, plusieurs associations recommandaient de dépister la maladie par un dosage de l'antigène spécifique prostatique chez les hommes âgés de 50 à 69 ans jusqu'à ce qu'on réalise que I'on avait oublié de faire la preuve de l'efficacité du dépistage; aujourd'hui, les recommandations déconseillent de prescrire cet examen. Certains diront que c'est parce que les preuves ont changé. $M$ ais, ce n'est pas toujours le cas. Prenons I'exemple du dépistage du diabète de type 2 : I'Association canadienne du diabèté nous recommande, actuellement, de dépister la maladie chez toutes les personnes âgées de plus de 45 ans et plus précocement, ou plus souvent en présence de facteurs de risque. 0 r, il n'a jamais été démontré que cette mesure changeait la morbidité ou la mortalité du diabète. L'Association le reconnaît implicitement puisqu'elle appuie cet avis seulement sur l'opinion d'experts. Se peut-il que dans quelques années cet avis change et, comme pour le cancer de la prostate, que les lignes directrices statuent qu'il n'est dorénavant plus nécessaire de procéder au dépistage?

Enfin, ce qui est probablement le plus préjudiciable aux lignes directrices, ce sont leurs incohérences, c'est-à-dire leur incapacitéà s'entendre les unes les autres sur un même sujet. Prenons l'exemple du dépistage du cancer du côlon. D epuis 1993, le dépistage au moyen del'H emoccut II suivi d'une côlonoscopie ou d'un lavement baryté permet de réduire de façon significative la mortalité de cette maladié. Or, face à cette preuve, deux grands organismes consultatifs jouissant d'une crédibilité internationale, le US Preventive Task Force et le Groupe de travail sur l'examen médical périodique ont réussi «l'exploit » d'émettre des recommandations diamétralement opposées : les Américains nous recommandant de procéder au dépistage du cancer du colon, les Canadiens jugeant que nousn'avions pas suffisamment de preuve pour recommander celui-ci !

En conclusion, les lignes directrices ne sont certainement pas aussi parfaites que nousl'imaginons. Souvent de piètre qualité, elles changent fréquemment d'avis et elles sont incohérentes entre elles mais surtout, elles sont, trop nombreuses. II n'est pas surprenant que, comme le démontrent plusieurs études, ${ }^{90}$, les médecins n'arrivent pas à suivre les recommandations des guides pratiques !

Alors, comment se fait-il que les médecins et les professionnels de la santé continuent à leur faire confiance et à les invoquer ? Peut-être tout simplement parce que confrontés à l'immensité des connai ssances médicales, les lignes directrices demeurent la seule solution possible. II est, cependant, grand temps que nous nous penchions sur le phénomène des lignes directrices et que nous développions des outils pour les évaluer de façon critique.

\section{Références}

1. Committee to Advise the Public H ealth Service on Clinical Practice Guiddines, Institute of M edicine. Field NJ, Lohr KN, eds. Clinical Practice Guidelines: Directons of a New Program. Washington, DC : N ational Academy Press; 1990.

2. BergAO, AtkinsD, TierneyW. Clinical practiceguidelinesin practice and education. J Gen Intern M ed $1997 ; 12$ (suppl 2) : S25-S33.

3. HibbleA, Kanada D, Pencheon D et coll. Guidelines in general practice: the now tower of Babel ? BMJ $1998 ; 317: 862-3$.

4. Shaneyfelt T, M ayo-Smith M F, Rothwangl J. Are guidelines following guidelines? T he M ethodogical Q uality of Clinical Practice Guidelines in the PerReviewed M edical Literature. JAM A $1999 ; 281$ (20) ; 1900-1905.

5. M eltzer S. et coll. 1998 clinical guidelines for the management of diabetes in Canada. CM AJ 1998 ; 159 (8 Suppl).

6. M andel JS, B ond JH, Church TR, Snover DS, Bradley GM , Schuman LM, Ederer F. Reducing mortality from col orectal cancer by screening for fecal occult blood. Colon Cancer Central Study. NEJM 1993 ; 328(19) : 1365-71.

7. U S Preventive ServicesTask Force Guide to dinical preventiveservices. 2nd ed. Baltimore: Williamsand Wilkins; 1996.

8. Groupe de travail sur l'examen médical périodique. Early detection of colorectal cancer. Can M ed Assoc) $1989 ; 141$ : 209-16.

9. Davis AD, Taylor-Vaisey A, Translating guidelines into practice. CM AJ 1997 ; 157 (4) : 408-16.

10. Cabana M D, Rand CS, PoweNR et coll. Why don't physiciansfollow dinical practiceguidelines?JAM A $1999 ; 282$ : 1458-65. 